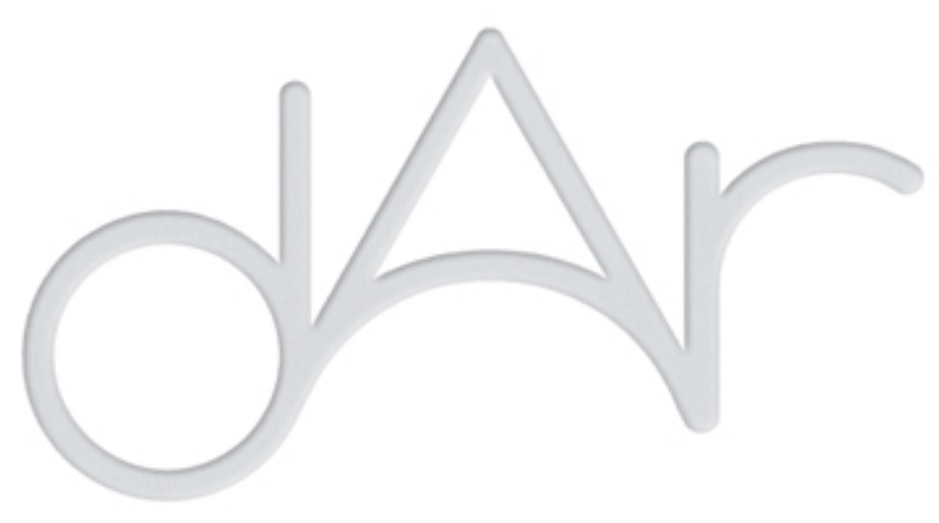

\title{
Quelles réflexions sur les retombées économiques du patrimoine: pour une valorisation des gravures rupestres d'Ait Ouaazik
}
Autor(es):
Faouzi, Mustapha
Publicado por: Imprensa da Universidade de Coimbra
URL
persistente: URI:http://hdl.handle.net/10316.2/44430
DOI: $\quad$ DOI:https://doi.org/10.14195/2182-844X_5_9
Accessed : $\quad$ 26-Apr-2023 16:33:08

A navegação consulta e descarregamento dos títulos inseridos nas Bibliotecas Digitais UC Digitalis, UC Pombalina e UC Impactum, pressupõem a aceitação plena e sem reservas dos Termos e Condições de Uso destas Bibliotecas Digitais, disponíveis em https://digitalis.uc.pt/pt-pt/termos.

Conforme exposto nos referidos Termos e Condições de Uso, o descarregamento de títulos de acesso restrito requer uma licença válida de autorização devendo o utilizador aceder ao(s) documento(s) a partir de um endereço de IP da instituição detentora da supramencionada licença.

Ao utilizador é apenas permitido o descarregamento para uso pessoal, pelo que o emprego do(s) título(s) descarregado(s) para outro fim, designadamente comercial, carece de autorização do respetivo autor ou editor da obra.

Na medida em que todas as obras da UC Digitalis se encontram protegidas pelo Código do Direito de Autor e Direitos Conexos e demais legislação aplicável, toda a cópia, parcial ou total, deste documento, nos casos em que é legalmente admitida, deverá conter ou fazer-se acompanhar por este aviso.

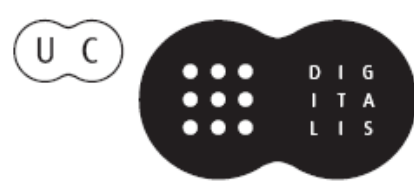



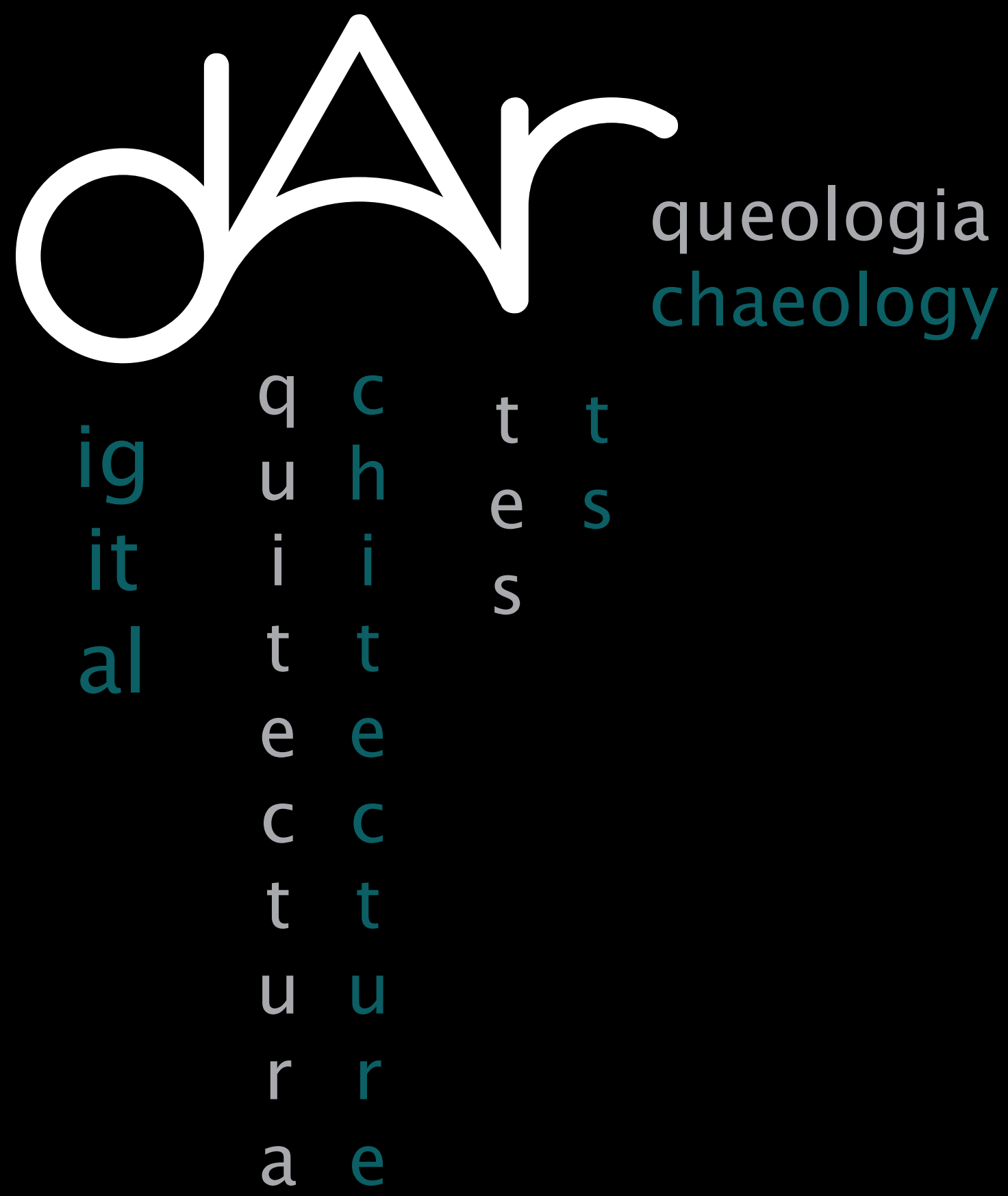

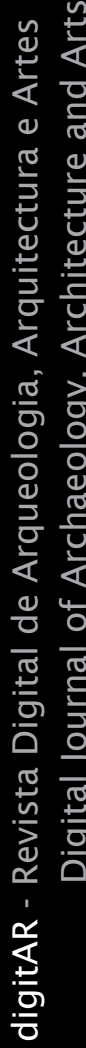




\title{
QUELLES RÉFLEXIONS SUR LES RETOMBÉES ÉCONOMIQUES DU PATRIMOINE : POUR UNE VALORISATION DES GRAVURES RUPESTRES D' AIT OUAAZIK
}

\author{
Dr. Mustapha Faouzi \\ Directeur de l'ANDZOA, Zagora
}

\section{Résumé}

Les gravures rupestres d'Aït Ouaazik sont parmi les plus connus du Sud marocain. Elles se trouvent à $70 \mathrm{~km}$ de route au nord de Zagora, région Draa Tafilalet (Maroc). Le village est situé dans une oasis dans le bassin de Maider, près de Tazzarine. Ces pétroglyphes dateraient du Néolithique. A cette époque la région Aït Ouaazik était une steppe..

Ces musées à ciel ouvert sont en grande partie méconnus, mais ceux dont le public a connaissance souffrent aujourd'hui de dégradations : climat et vandalisme. «Les aires rupestres marocaines sont d'accès libre et aucun droit de visite n'est acquitté par personne.

Ce patrimoine aura des retombées économiques directes et indirectes s'il est valorisé. Et par conséquent, l'amélioration des conditions de vie de la population locale qui sera prise en compte dans l'exploitation et la valorisation du site. En effet, s'approprier le patrimoine par sauvegarde d'abord, la réhabilitation et également le plaidoyer pour son rayonnement.

$C^{\prime}$ est dans ce sens que l'Agence nationale pour le développement des zones oasiennes et de l'arganier (ANDZOA), dans le cadre du projet d'adaptation aux changements climatiques (PACCZO) compte avec tous les acteurs locaux, à entamer des mesures pour réhabiliter ce site afin de drainer des ressources à cette localité en soutenant des initiatives locales au profit des jeunes et des femmes dans les secteurs de l'agriculture, le tourisme, la culture et l'artisanat.

\section{Introduction}

Le patrimoine est encore trop souvent perçu comme une charge, que des sociétés riches peuvent à la rigueur assumer par prestige, mais coûteuse 
et sans valeur économique.

À partir de cette idée reçue, la tentation est grande de tailler dans un budget considérécommeunluxeinutile, dans des périodes difficileséconomiquement et financièrement où il faut éliminer les dépenses superflues.

Loin de faire du patrimoine, par une petite élite socioculturelle, un effet de mode et le galvauder dans les salons sans avoir un effet concret sur le bien-être de la population, il faut ancrer l'importance du patrimoine dans le développement mais également l'enraciner dans une mutation profonde et durable des sensibilités sociétales.

$\mathrm{Au}$ temps de la mondialisation qui donne le sentiment de l'uniformité et entraîne une perte des repères, la contemplation de témoins d'un passé plus ou moins lointain rassure et paraît une compensation à un avenir incertain. Il existe aujourd'hui de multiples formes de cette nostalgie d'anciens temps disparus. Mais les monuments inscrits dans des paysages contemporains ont une puissance d'évocation sans commune mesure et suscitent le rêve et l'imagination, l'un des attraits les plus puissants du tourisme patrimonial.

\section{Le patrimoine, qu'est ce que c'est ? C'est un concept élargi}

Chaque nation a son patrimoine qui lui a été transmis de génération en génération. Il s'agit là d'un héritage commun qui définit l'identité de toute une communauté. Le patrimoine nous définit en tant qu'individu appartenant à un groupe social. Il est le témoin de l'évolution de chaque société. C'est grâce à lui que nous pouvons connaître l'histoire de notre pays et comprendre le fonctionnement actuel de notre société. Ce patrimoine peut être des monuments, des objets et même des savoirs faires. En définitive, le patrimoine c'est ce qu'ont fait nos ancêtres et qui nous sert à comprendre pourquoi nous vivons ainsi aujourd'hui. C'est pourquoi il existe plusieurs types de patrimoines, différents en apparence, mais qui s'inscrivent tous dans notre passé commun.

L'Unesco le définit comme "l'héritage du passé, dont nous profitons aujourd'hui et que nous transmettons aux générations à venir » (conférence de 1972).

\section{L'appréhension économique du patrimoine}

Le patrimoine a été considéré comme un outil de conservation, puis comme un élément essentiel de constitution de la nation (CHOAY, 1988 ; POULOT, 2006), le patrimoine est devenu ressource pour la construction et le 
développement des territoires. Cette nouvelle situation pose alors la question des enjeux, des formes et des logiques de cette prolifération patrimoniale au regard des dynamiques territoriales.

Pour être durablement sauvegardé, le patrimoine doit avoir une fonction, répondre à un besoin contemporain. La valorisation du patrimoine s'inscrit donc désormais dans une perspective de politique intégrée, d'attractivité et de dynamisation des territoires. La prise de conscience du rôle économique significatif joué par le patrimoine et la nécessité d'en mesurer l'impact se renforce : on passe du souci de maintenir la "source de la rente » à une démarche dynamique de production de services fortement territorialisée, articulant des dimensions culturelles, historiques, scientifiques, sociales, économiques. Par conséquent, on peut avancer que le patrimoine peut être engagé dans la construction d'un territoire.

L'hypothèse que nous proposons repose sur l'idée que le patrimoine constitue une dimension essentielle de la ressource territoriale et que sa mobilisation traduit l'émergence d'un mode de développement territorial spécifique.

Pour aborder cette réflexion, notre démarche reposera sur un diagnostic territorial d'un bassin de vie appelé « le maider » comportant cinq communes territoriales ( à savoir : la commune territoriale de Tazarine, la CT de Nkob, la CT de Taghbalte, la CT de Ait Bouadoud et la CT de Ait Ouallal). Ce territoire oasien peut articuler son projet de développement alternatif sur le patrimoine dont il regorge : tourisme cultuel (18 marabouts), et tourisme patrimonial : paléontologique et géologique, archéologique et autres.

Dans ce contexte, la mesure et la prise en compte de l'impact économique et social du patrimoine représentent un enjeu déterminant pour les différents acteurs (institutionnels, associatifs, économiques, scientifiques), qui interviennent dans sa gestion, sa restauration, sa conservation et sa valorisation.

\section{Les retombées économiques du patrimoine}

Greffe Xavier défini le terme " retombées » comme un ensemble d'effets économiques, sociaux, fiscaux ou en termes d'investissements publics et d'attractivités observables sur un territoire donné et liés à la présence d'un site patrimonial.

Le développement d'un territoire peut se faire sur une mythologie c'est pour cela, il nous semble pertinent de poser la question de l'émergence d'un mode de développement alternatif, en rupture avec le mode de développement où la productivité caractérisait la compétitivité et où l'innovation était exogène 
au territoire.

Il faut démontrer que loin de coûter, le patrimoine protégé est une source importante d'emplois et de revenus. Dans ce contexte, la mesure et la prise en compte de l'impact économique et social du patrimoine représentent un enjeu déterminant pour les différents acteurs (institutionnels, associatifs, économiques, scientifiques), qui interviennent dans sa gestion, sa restauration, sa conservation et sa valorisation. Cette réflexion permet de repérer les différentes formes d'impact du patrimoine, les potentialités régionales, et la nature précise des retombées directes. Plus largement, on peut attester à quel point, loin d'être une charge, le patrimoine est porteur d'une dynamique économique conséquente. Ceci est de nature à aider les institutions concernées et les acteurs du patrimoine à valoriser les projets et actions conduits, à s'engager dans des politiques de valorisation ambitieuses et efficientes.

\section{a. Les retombées directes du patrimoine}

Elles sont définies comme des retombées (qui agissent sur la structure patrimoniale ou qui émanent directement d'elle) un site patrimonial à entrée payante constitue un pôle économique produisant un chiffre d'affaires, une valeur ajoutée, des emplois, des recettes fiscales. Ces éléments constituent les retombées directes du patrimoine.

On peut citer succinctement :

L'aménagement du site lui-même, l'accessibilité au site ;

* les emplois directs du patrimoine, mobilisés par le monument : billetterie et visite guidée. Ces personnes ont en charge la préservation, la conservation, la gestion, la mise en valeur et l'accessibilité des publics ;

* les recettes provenant de l'exploitation d'une boutique-librairie à travers les dérivés du patrimoine.

\section{b. Les retombées indirectes du patrimoine}

Les visiteurs d'un site patrimonial effectuent des dépenses dans l'environnement immédiat du site patrimonial (par exemple dans le secteur hôtelier, dans les commerces liés au site ou à l'artisanat local...). Ces dépenses alimentent le chiffre d'affaires des agents chez qui ces dépenses sont effectuées, générant une valeur ajoutée, des emplois, des recettes fiscales. Par retombées sociales indirectes, nous entendons les effets du patrimoine en termes d'emplois dans des secteurs fournissant des biens et des services 
au secteur du patrimoine. Entrent dans cette catégorie les emplois liés à la restauration du patrimoine, à l'exploitation touristique du patrimoine, et aux autres emplois culturels liés à la valorisation du patrimoine : formation et recherche, artisanat d'art et expertise.

\section{Quelles réflexions pour la valorisation des gravures rupestres d'AIT OUAAZIK}

\section{Constat de l'art rupestre au Maroc}

L'art rupestre constitue une richesse artistique, culturelle, archéologique et scientifique irremplaçable, mais il est dans une situation de précarité et par conséquent, il encoure le risque de la disparition. L'on sait que la disparition d'un aspect patrimonial est naturellement irréversible et le sera ainsi à jamais comme le souligne Mustapha NAMI, archéologue du ministère de la culture ${ }^{1}$.

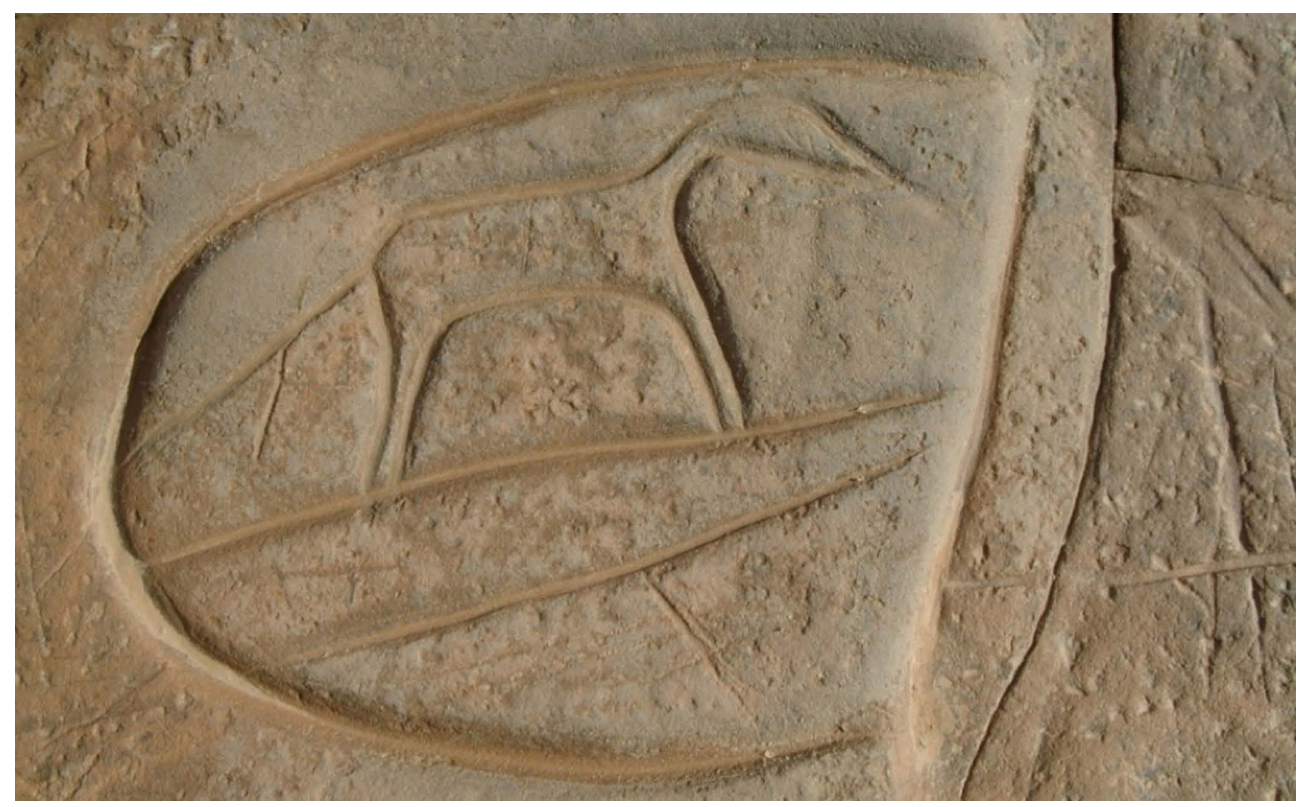

Photo 1 - Art rupestre dans la zone. Source : CERKAS

1 Note sur les gravures rupestres au Maroc, rédigée par Mustapha NAMI du ministère de la cultureDirection du patrimoine le 7avril 2017 
L'état de conservation des sites rupestres au Maroc laisse à désirer. L'érosion et la dégradation naturelle ainsi que le vandalisme anthropique sont les menaces potentielles des gravures. Si le premier facteur a toujours été présent et agit d'une manière très lente, le deuxième facteur, par contre, constitue la source d'une inquiétude croissante tant ses effets ne cessent de s'intensifier d'une manière exponentielle, dénote toujours ce chercheur qui ne cesse de sonner le tocsin d'alarme. Le constat est en effet alarmant quand on sait que, quotidiennement, des dalles portant des figurations rupestres disparaissent à jamais. Les exemples ne manquent pas : des sites entiers ont complètement disparu et ne font plus partie de la carte archéologique du pays. Les causes et les finalités de cette disparition sont multiples, mais elles se déclinent en deux aspects majeurs :

- Le premier est relatif à une dévastation consciente et fait partie du problème funeste du trafic illicite et du pillage des biens culturel, dont le Maroc souffre cruellement et plus particulièrement au Présahara, au Haut-Atlas et dans l'Anti-Atlas. Le développement de ce genre de trafic n'a pas en effet, épargné les grands chefsd'œuvre de l'art rupestre marocain. De belles images rupestres ont effectivement été affreusement arrachées de leurs contextes et emportées pour décorer des jardins privés, et ce, depuis l'époque des colons.

- $\quad$ Le deuxième aspect est quant à lui qualifié d'une destruction inconsciente en ce sens que les activités destructives ignorent complètement l'existence et/ou l'importance de telles images gravées ou peintes.

$\mathrm{D}^{\prime}$ autres formes de dégradation anthropique sont également relevables dans la plupart des sites rupestres du Sud marocain. Ceci concerne par exemple le problème du repassage des traits gravés par des touristes mal avertis ou peu consciencieux qui n'hésitent pas de procéder à ce type d'actions pour mieux mettre en relief les images gravées peu visibles et réussir ainsi leurs photos! D'autres antiquaires clandestins ou trafiquants d'objets patrimoniaux font appel à la technique du moulage en utilisant malheureusement des produits non appropriés et qui affectent sérieusement le trait et la patine de la figure. Dans des cas extrêmes, les supports rocheux s'effritent aussitôt à la suite de l'utilisation de tels produits.

Une prise de conscience collective s'impose pour ainsi protéger cet héritage ancestral pour le transmettre aux générations futures. La réappropriation du patrimoine culturel en tant que reflet d'une identité historico-culturelle profonde serait le catalyseur d'une telle prise de conscience. 


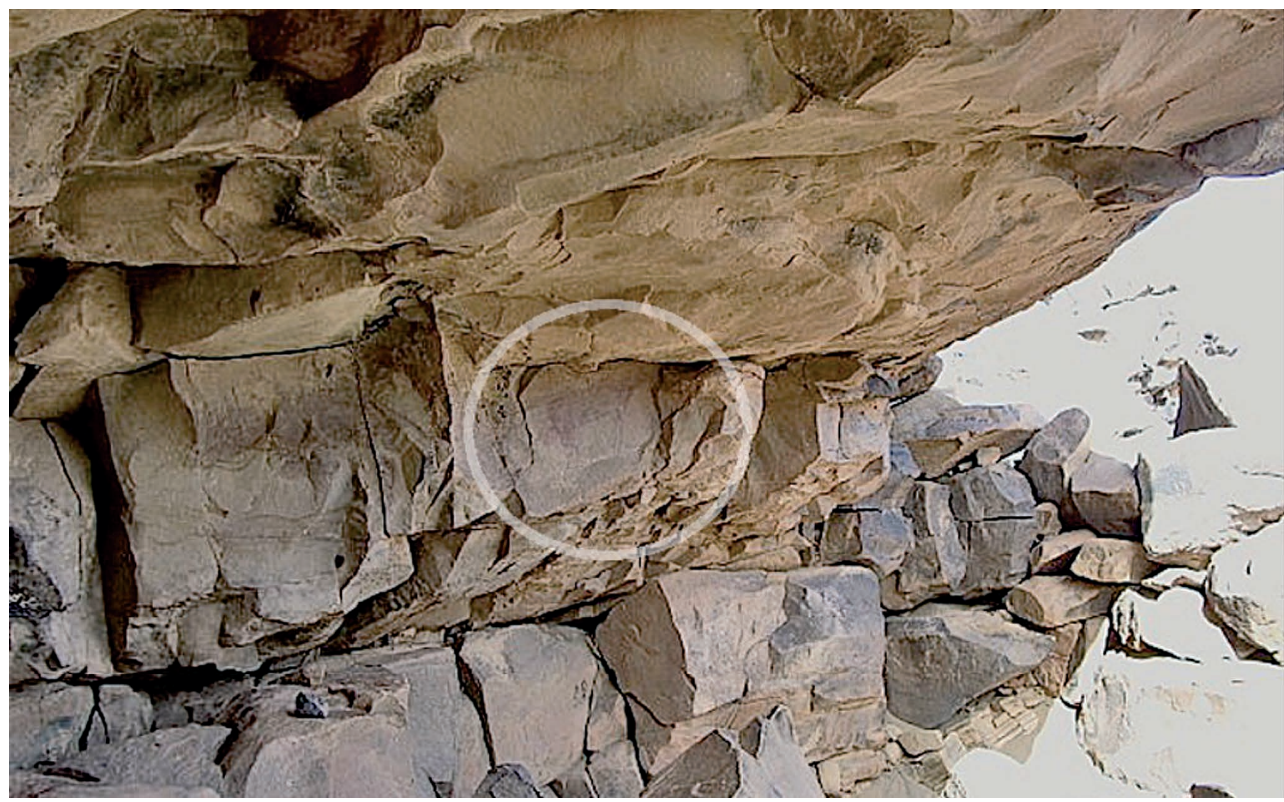

Photo.2 : Foum d'Imi n'Taroucht. Source : Lafanachère en 1953

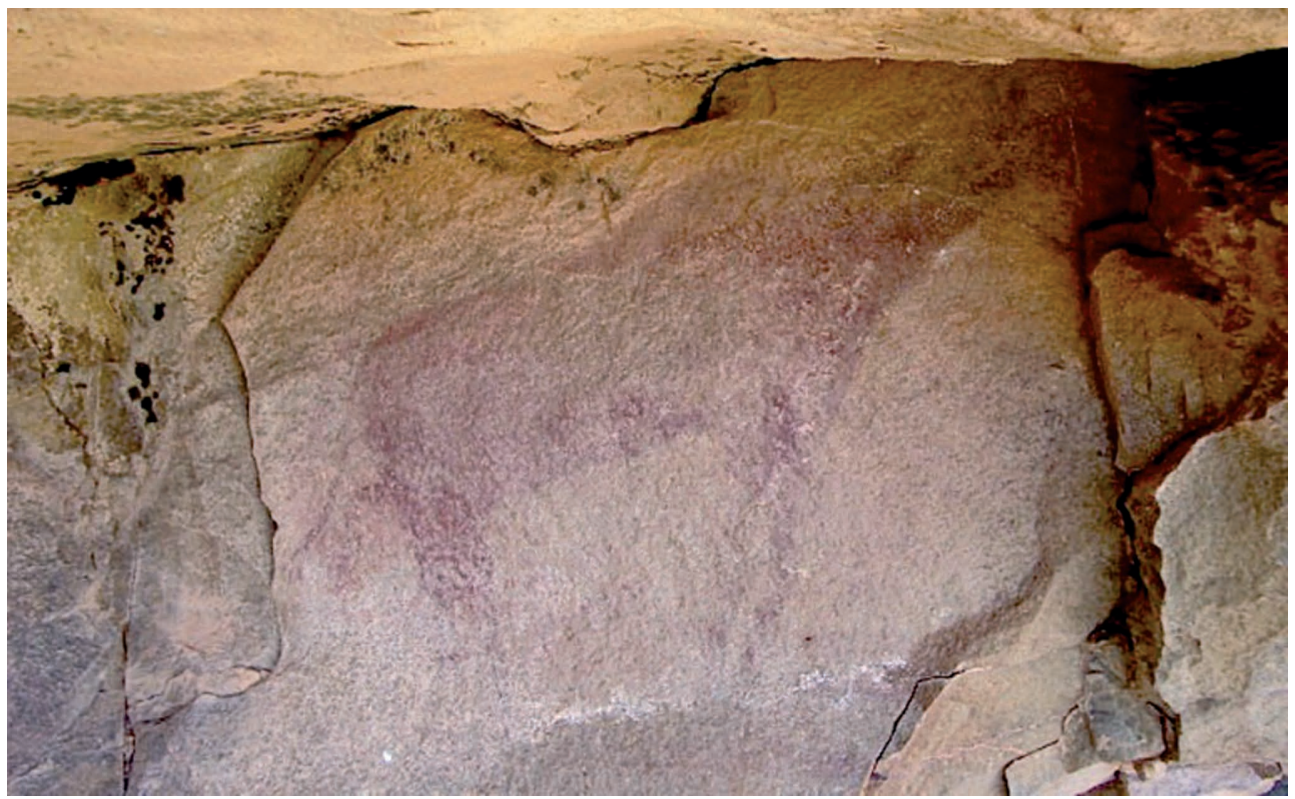

Photo.3 : Foum d'Imi n’Taroucht 


\section{Les gravures rupestres d'Ait Ouaazik}

Situé au Sud-Est du Maroc, la vallée de Draâ à Zagora présente l'une des régions les plus riches en gravures rupestres. Sur les 300 sites rupestres marocains, le Draâ moyen renferme une vingtaine. Ils sont éparpillés sur l'ensemble du territoire et plus particulièrement au niveau des zones de Maîder (Tazarine), et le long d'oued Draa : Tinzouline, Tamgroute, Tagounite. Pour conserver et réhabiliter ce patrimoine de la province, un projet d'une grande envergure est lancé par le Centre de conservation et de réhabilitation du patrimoine architecturel des zones atlassiques et subatlassiques (CERKAS) à travers l'initiative de ministère de la Culture, direction du patrimoine culturel. Il s'agit du projet de l'aménagement des sites des gravures rupestres dans la vallée de Draâ et la vallée de Maîder, plus exactement sur le site de Foum Chenna (commune Tinzouline) et le site Aït Ouaazik (commune de Tazarine). En vue de protéger et valoriser les gravures rupestres, les deux sites dont Foum Chenna et Ait Ouaazik seront protégés par l'aménagement et la construction de gabions, outre l'aménagement d'un passage touristique, et la construction d'une salle d'exposition et de la maison du gardien du site qui va veiller sur ces gravures. Le projet de l'aménagement des sites des gravures rupestres de Foum Chenna et d'Aït Ouaazik visent non seulement la protection et la valorisation des gravures rupestres, une composante patrimoniale d' une valeur inestimable, mais aussi l'encouragement de l'activité touristique dans la vallée du Draâ et de Maîder et de la recherche scientifique par des missions d'études et de recherches. Ces réalisations vont encourager le tourisme culturel et artistique, permettre également aux étudiants, chercheurs de différentes horizons, de venir dans les sites pour approfondir leurs études. Notons de passage que les gravures rupestres présentent une grande importance car elles reflètent la civilisation millénaire de la région en particulier et du Maroc en général. ${ }^{2}$

En effet, les gravures rupestres anciennes remontent à 5000 av JC, d'un temps où le Sahara était peuplé d'animaux sauvages rhinocéros, éléphants, rhinocéros, girafes, autruches, antilopes, cheval, gazelles... Le site est un témoignage d'un temps, où le Sahara était vert et rempli d'animaux maintenant bien éloignés, comme l'éléphant ou la girafe. Et l'antilope à immenses cornes, sans doute un ancêtre de l'oryx ou de l'hippotragus, qu'on ne trouve maintenant qu'en Afrique centrale. Ainsi sont des vestiges et des

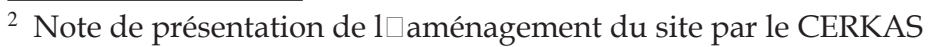


indices de l'existence humaine dans la région.

Cette réflexion va contribuer non seulement à la protection du site mais aussi à l'amélioration des conditions de vie de la population locale qui sera prise en compte dans l'exploitation et la valorisation du site. Mais, malgré ces potentialités, le site rencontre des problèmes de protection; d'où sa dégradation. Ce constat nous a amené à faire une proposition de contribution à la protection du patrimoine culturel et à la gestion de son environnement. Etant donné que la culture est un vecteur constituant un élément d'identification d'un peuple, sa valorisation doit tenir compte de l'environnement. De ce fait, nous pouvons dire que la protection du patrimoine culturel doit supposer celle de l'environnement. En effet, les deux actions doivent être liées pour profiter aux générations futures; ce qui permet d'intégrer le développement durable dans notre démarche. $\mathrm{Si}$ le développement durable est « un développement qui répond aux besoins des générations du présent sans compromettre la capacité des générations futures à répondre aux leurs » (Rapport Brundtland de 1987), nous devons faire intervenir et mettre en avant la capacité de mieux gérer le patrimoine culturel en vue de le de transmettre par la suite.
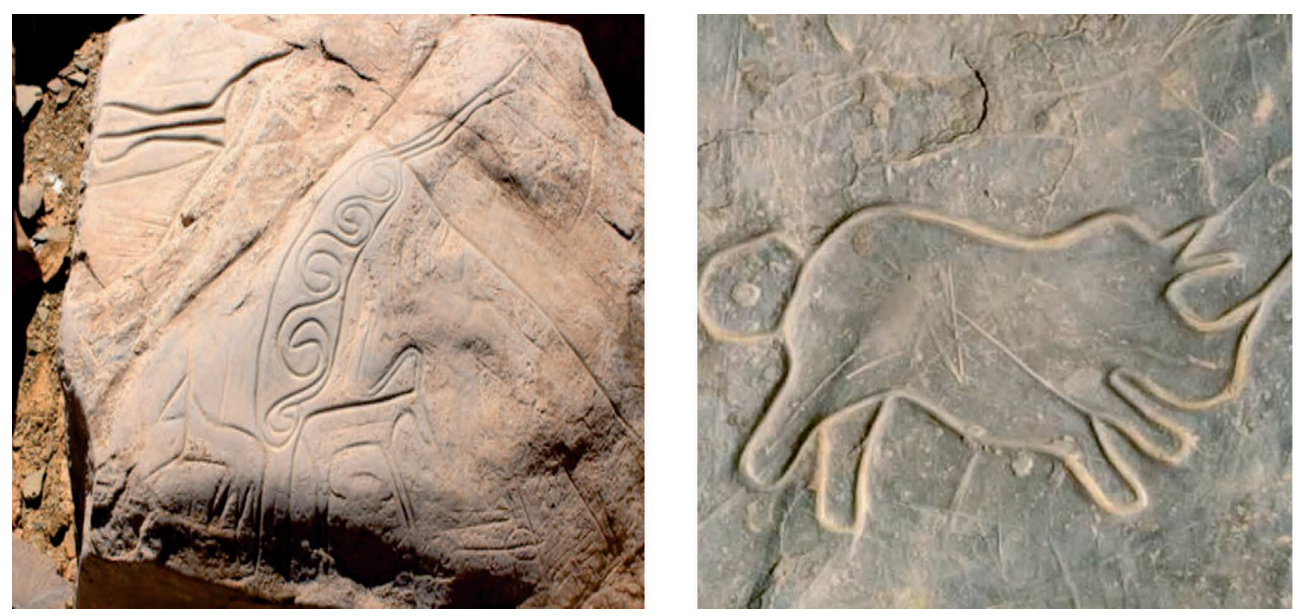

Photo 4 - Richesse du patrimoine archéologique dans la zone

En réalité, nous sommes partis du fait selon lequel le patrimoine culturel est une richesse et une richesse exploitée à bon escient entraine un développement des conditions de vie de la population locale. C'est pourquoi, le père de la 
négritude, Léopold Sédar Senghor disait « la culture est au commencement et à la fin du développement ».

Nous focaliserons nos propositions sur le site Ait Ouaazik, mais il est important de savoir que cet exemple peut être appliqué aux autres sites, dont regorge la région. A cet effet, une association de préservation de ce patrimoine est créée dans la commune de Tazarine pour sensibiliser les acteurs locaux de l'importance de cette richesse afin de s'approprier ce patrimoine en le sauvegardant d'abord, le réhabiliter et également plaidoyer pour son rayonnement.

C'est dans ce sens que l'Agence nationale pour le développement des zones oasiennes et de l'arganier, dans le cadre du projet d'adaptation aux changements climatiques (PACCZO) compte avec tous les acteurs locaux (population, société civile, commune territoriale et autres), à œuvrer avec le département de la culture, via le CERKAS, à entamer des mesures pour réhabiliter ce site afin de drainer des ressources à cette localité puisque ce projet prévoit le soutien des initiatives locales au profit des jeunes et des femmes dans les secteurs de l'agriculture, le tourisme, la culture et l'artisanat. Outre l'existant déjà élaboré et construit par le CERKAS, des mesures à entreprendre dans le cadre d'un partenariat sont comme suit :

- Aménagement du site objet des gravures rupestres : aménagement du chemin pédestre ;

- veiller à l'élaboration d'un plan de protection et de réhabilitation de site pour éviter des gribouillis, écrits, graffitis et peintures, pour ne pas altérer ce legs archéologique et humanitaire, et éviter également les actes de vandalisme ayant affecté ce musée à ciel ouvert ;

- fourniture des panneaux signalétiques dans différents axes routiers ;

- meubler la salle d'exposition ;

- équipement en solaire de la salle d'exposition avec des dépendances sanitaires,

- rampes d'accès pour handicapés,

- production de film documentaire pour promouvoir le tourisme dans la région afin de générer des ressources à la commune territoriale ;

- veiller à inscrire ce bien comme patrimoine national voire international ;

- élaborer une stratégie de gestion des visiteurs en construisant des plateformes ou passerelles en bois pour protéger les gravures de toute dégradation et laisser les gravures sur leur soubassement rocheux;

- développer les dérivés du patrimoine pour la promotion et générer 
également des revenus et des ressources : boutique- cadeaux, comportant souvenirs (cartes postales, portes clés, produits artisanaux, etc.)Les clients des boutiques de sites cherchent à prolonger ou à partager l'expérience vécue lors de la visite, en acquérant des objets à valeur émotive. Les boutiques, à l'image des lieux qui les accueillent, doivent monter en gamme dans l'offre proposée et dans l'agencement de l'espace.

\section{Conclusion}

Le patrimoine peut s'affirmer comme un marché majeur de l'activité économique et touristique pour ce territoire. C'est un vrai levier de développement, s'il est bien exploité par l'ensemble des acteurs par le biais de leur adhésion et leur appropriation. Certes, c'est une initiative timide, mais je crois que c'est le premier pas dans un long parcours pour faire du patrimoine, voire de la culture, le vrai challenge de mutations de ces territoires.

\section{Bibliographie}

Choay (F) 1992 : L'allégorie du patrimoine. Édition Seuil. Paris 1992. 272 pages.

Rodrigue,(A), 2009 : L'art rupestre au Maroc : les sites principaux, des pasteurs du Dra aux métallurgistes de l'Atlas, L'harmattan.

Jean- Luc-PIERRE, 2012 : Quand les anciens priaient pour la pluie p78-81, ZAMANE No19 MAI,2012

Greffe, Xavier, 2003: La valorisation économique du patrimoine, la Documentation française.

Pierre-Antoine Landel \& Nicolas Senil, 2009: Patrimoine \& territoire, les nouvelles ressources du développement, revue développement durable, dossier $\mathrm{N}^{\circ} 12$.

Jean-Michel Kosianski, 2011 : Territoire, culture et politiques de développement économique local : une approche par les métiers d'art, in :Revue d'Économie Régionale \& Urbaine. 